\title{
Tratamiento del dolor: técnicas locorregionales. Bloqueos
}

M. S. Acedo Gutiérrez

\section{Consideraciones generales}

Un tercio de los pacientes con una neoplasia presentan dolor en un "estadio intermedio" de su enfermedad y aproximadamente un $70-80 \%$ lo presentan en un estadio avanzado. La incidencia del dolor está en función del tipo de neoplasia primaria, siendo la incidencia mayor en los tumores óseos primarios, tumores urológicos y de mama.

Entre un $70-90 \%$ del dolor producido por cáncer puede ser controlado con medicación oral, pero hay un $10-30 \%$ de los casos en los que va a ser necesario el uso de procedimientos invasivos para controlar el dolor, entre ellos las técnicas locorregionales. Es importante tener en cuenta que las técnicas locorregionales en el manejo del dolor de origen neoplásico se utilizan como tratamiento coadyuvante analgésico, y nunca como un tratamiento definitivo. Estos procedimientos van a permitir disminuir las dosis de fármacos, reducir los efectos secundarios y mejorar así la calidad de vida.

El abordaje terapéutico del dolor va ser siempre multidisciplinar y multimodal, pudiéndose combinar el tratamiento fármacológico oral, el tratamiento etiológico, las técnicas invasivas, las medidas físico-ortopédicas, las técnicas psicológicas cognitivo-conductuales, la cirugía paliativa...cuyos objetivos comunes son procurar una mayor calidad de vida al paciente. A grandes rasgos dentro de las técnicas intervencionistas para el tratamiento del dolor oncológico se pueden considerar: 1) Bloqueos nerviosos, que se realizan sobre el sistema nervioso autónomo, el sistema nervioso periférico y el neuroeje; 2) Infusión de fármacos en el sistema nervioso central (cloruro mórfico, anestésicos locales, clonidina); y 3) Técnicas neuroquirúrgicas ablativas (rizotomía, cordotomía percutánea, hipofisectomía,...). En este texto se pretende describir el uso de los bloqueos nerviosos en el tratamiento del dolor crónico oncológico.

Unidad Multidisciplinaria para el Estudio y Tratamiento del Dolor

Hospital Universitario 12 de Octubre

Madrid

\section{Bloqueos analgésicos}

\section{Fundamentos clínicos}

El objetivo al realizar un bloqueo nervioso es denervar áreas dolorosas y/o interrumpir las vías dolorosas del sistema nervioso central que conducen las aferencias nociceptivas al cerebro. Así se consigue eliminar un foco de irritación nociceptiva e interrumpir la percepción del dolor. Por otro lado también puede interrumpir el componente aferente de los mecanismos reflejos anormales, eliminando las respuestas reflejas, así como interrumpir la conducción de los impulsos simpáticos eferentes.

\section{Indicaciones de los bloqueos nerviosos}

Los bloqueos nerviosos pueden realizarse con fines diagnósticos, pronósticos y terapéuticos, o una combinación de ellos.

A) Bloqueos diagnósticos. Son útiles para conseguir información acerca de los mecanismos del dolor. Sirven para identificar el origen anatómico del dolor, identificar las vías nociceptivas, diferenciar entre el dolor local y el referido y precisar la contribución del sistema nervioso simpático en el cuadro doloroso. Son de ayuda a la hora de realizar el diagnóstico diferencial en cuadros de sintomatología confusa, distinguiendo entre dolor somático o visceral, o entre dolor periférico o central.

B) Bloqueos pronósticos. Ayudan a predecir el efecto de una intervención neuroquirúrgica o un bloqueo neurolítico. Proporcionan al paciente la oportunidad de experimentar los efectos posibles de una operación o un bloqueo neurolítico, como son la analgesia que puede obtener, las sensaciones anormales que puede presentar (parestesias, disestesias, anestesia) y otros efectos que puedan suceder. Se facilita así la decisión del paciente y una mejor selección de los casos.

C) Bloqueos terapéuticos. Se pueden realizar bloqueos temporales con anestésicos locales o bloqueos prolongados con agentes neurolíticos. Con los bloqueos temporales se puede conseguir alivio completo del dolor por un periodo de tiempo limitado, interrumpir los reflejos anormales rompiendo el círculo vicioso del dolor, así como la sintomatología simpática, facilitándose el empleo de otras terapéuticas (como la rehabilitación). Los bloqueos con agentes neurolíticos (alcohol, fenol) están limitados principalmente para el trata- 
TABLA I

\section{Bloqueos nerviosos espinales}

\begin{tabular}{ll}
\hline Bloqueo & Indicación \\
\hline A.- Cervicales & \\
1.- Occipital & Neuralgia \\
2.- Plexo cervical & Dolor y malignidad \\
3.- Frénico & Hipo rebelde, dolor diafragmático \\
4.- Plexo braquial & Dolor y malignidad \\
5.- Supraescapular & Periartritis \\
& \\
B.- Torácicos & Herpes zoster/Neuralgia \\
1.- Paravertebral & Malignidad \\
& Cicatriz dolorosa postoracotomía \\
2.- Intercostal & Metástasis en costilla \\
& \\
C.- Lumbares y sacros & Neuralgia \\
1.- Paravertebral & Diagnóstico/terapeútico \\
2.- Trans-sacro & Dolor en ingle \\
3.- llioinguinal & Meralgia parestésica \\
4.- Femorocutaneo & Previo a neurectomía \\
5.- Obturador & Neuralgia \\
6.- Femoral y ciático & \\
\hline
\end{tabular}

TABLA ॥

\section{Bloqueos de pares craneales}

\begin{tabular}{ll}
\hline Bloqueo & Indicación \\
\hline $\begin{array}{l}\text { Trigémino } \\
\text { Oftálmico } \\
\text { Maxilar, mandibular }\end{array}$ & $\begin{array}{l}\text { Tic doloroso, dolor en cáncer } \\
\text { Dolor ocular (glaucoma, uveitis) } \\
\text { Tic doloroso, cáncer }\end{array}$ \\
Facial & Espasmo facial \\
Glosofaringeo & Tic doloroso, dolor a la deglución \\
Vago & Broncoscopia y terapia de reflejos \\
& vagales
\end{tabular}

miento del dolor de origen oncológico en la fase avanzada de la enfermedad. El objetivo de este bloqueo es destruir la fibra nerviosa y producir así un bloqueo prolongado y a la vez permanente similar al que se produciría con una sección quirúrgica.

\section{Tipos de bloqueos nerviosos}

Según el nivel al que se realice el bloqueo se pueden clasificar en primer lugar los que actúan sobre el sistema nervioso periférico, en segundo lugar sobre el sistema nervioso autónomo y en tercer lugar sobre el neuroeje.

A) Bloqueos nerviosos periféricos. Es bien sabido que cualquier nervio periférico tanto craneal como espinal, puede ser abordado con una aguja interrumpiendo su función.
El bloqueo con agentes neurolíticos (alcohol, fenol) de los nervios periféricos puede ser eficaz como analgesia coadyuvante al tratamiento oral; no obstante son técnicas cada vez más restringidas. Los bloqueos periféricos más comunes y su utilidad en diversas patologías dolorosas se reflejan en las Tablas I y II.

B) Bloqueos sobre el sistema nervioso autónomo: bloqueos simpáticos. El sistema nervioso autónomo es en gran parte responsable de la nocicepción visceral. Un bloqueo diagnóstico con anestésico local sobre el sistema simpático o plexo correspondiente establece la relativa contribución del dolor visceral y el sistema autónomo y reproduce el efecto que se podría conseguir con un bloqueo neurolítico. En la Tabla III se presentan los diferentes tipos de bloqueos simpáticos y su aplicación en cuadros de dolor. De especial mención por su aplicación en dolor oncológico es el bloqueo del plexo celíaco, también denominado plexo solar o esplácnico. Es el más largo de los tres grandes plexos del sistema nervioso simpático en el tórax y abdomen; el plexo cardiaco inerva las estructuras torácicas, el plexo celíaco los órganos abdominales y el plexo hipogástrico los órganos pélvicos. El plexo celíaco inerva la mayor parte de las visceras abdominales incluyendo estómago, hígado, suprarrenales, epiplon, intestino delgado y grueso hasta ángulo esplénico. Se utiliza fundamentalmente para tratar el dolor producido a nivel abdominal por el cáncer de páncreas. Se sitúa en la región anterolateral ala aorta a nivel de las vértebras T12-L2. El bloqueo del ganglio celíaco con alcohol alivia el dolor con una eficacia superior al $80 \%$ en el dolor de origen visceral intraabdominal.

\section{TABLA III}

\section{Bloqueos simpáticos}

\begin{tabular}{ll}
\hline Bloqueo & Indicación \\
\hline Ganglio estrellado & Dolor en cabeza y brazo \\
Ganglio de Gasser & $\begin{array}{l}\text { Neuralgia del trigémino y } \\
\text { dolor facial }\end{array}$ \\
$\begin{array}{l}\text { Interpleural (torácico } \\
\text { y cadena simpática) }\end{array}$ & $\begin{array}{l}\text { Alto-cabeza y brazo } \\
\text { Bajo-vejiga, órganos abd. y útero }\end{array}$ \\
Plexo celiaco & $\begin{array}{l}\text { Pancreatitis, dolor abd., dolor } \\
\text { (nervios esplácnicos) }\end{array}$ \\
Simpático lumbar & Miembro fantasma doloroso \\
Plexo hipogástrico & $\begin{array}{l}\text { Perineal, pélvico, miembro } \\
\text { fantasma doloroso }\end{array}$ \\
Glanglio sacrocoxigeo (Walther) & Dolor rectal
\end{tabular}

C) Bloqueos raquideos (neuroeje). Con el objeto de conseguir alivio duradero, la destrucción del impulso nervioso debe realizarse a nivel preganglionar, lo cual se consigue por medio de la inyección intratecal de fármacos neurolíticos. Se 


\section{S. Acedo Gutiérrez}

puede realizar a nivel intra- o extradural. La inyección del agente neurolítico, alcohol o fenol, debe realizarse donde los nervios salen del canal raquideo a través del agujero intervertebral. El paciente puede experimentar sensaciones de dolor urente o parestesias desagradables durante los primeros segundos y luego aparece analgesia completa. Estos bloqueos se han dejado de utilizar por el alivio temporal, resultados poco satisfactorios, importantes efectos secundarios (trastorno de esfínteres, mielitis transversa) desde la aparición de nuevos fármacos y nuevas vias de administración que minimizan los efectos secundarios.

\section{Requisitos para obtener óptimos resultados con los bloque- os nerviosos}

Los requisitos para obtener óptimos resultados con los bloqueos nerviosos son:

1. Tener interés en los problemas dolorosos y un conocimiento completo de los síntomas y posibles mecanismos de producción.

2. Actuar como médico y no como técnico, realizando una historia clínica completa, confirmando o rechazando el diagnóstico y dedicando el tiempo y esfuerzo necesario.

3. Tener habilidad y maestría con las diferentes técnicas de bloqueos, para lo cual se debe tener un conocimiento de la anatomía, farmacología y de las posibles complicaciones y su tratamiento. Es necesario mantener la habilidad técnica con enfermos quirúrgicos.

4. Es muy importante sentar la indicación correcta del bloqueo.

\section{Bibliografía}

Vicente Fatela L. Técnicas invasivas en el tratamiento del dolor neoplásico. Rev. Cancer. 2000; 14: 208-217.

Madrid Arias J.L. Evolución histórica de los agentes neurolíticos utilizados para tratamiento del dolor crónico. Rev. Esp. Anest.-Ream. 1984; 31 (5): 197-204.

Ferrer-Brechner T. Anesthetic for the manegement of cancer pain. Cancer 1989; 63: 2343-2347.

Swerdlow M. Neurolytic blocks of the neuraxis. En: Patt R.B. (ed.). Cancer pain. J.B. Lippincott Company, Philadelphia, 1993; 427444.

Fitzgibbon D.R. Dolor por cancer: tratamiento. En: Loeser J.D. (ed). Bonica Terapéutica del Dolor. 3a ed. Mexico: McGraw-Hill Interamericana; 2003. p. 815-28.

Buckley F.P. Anestesia regional con anestésicos locales. En: Loeser J.D. (ed). Bonica Terapéutica del Dolor. $3^{\underline{a}}$ ed. Mexico: McGrawHill Interamericana; 2003. p. 2247-2320.

Butler S.H., Charlton J. E. Bloqueo con neurolíticos e hipofisectomía. En: Loeser J.D. (ed). Bonica Terapéutica del Dolor. $3^{\mathrm{a}}$ ed. Mexico: McGraw-Hill Interamericana; 2003. p. 2338-86. 